\title{
Beneficial Effects of 6-Month Supplementation with Omega-3 Acids on Selected Inflammatory Markers in Patients with Chronic Kidney Disease Stages 1-3
}

\author{
Agnieszka Pluta, ${ }^{1}$ Pawel Stróżecki, ${ }^{2}$ Jacek Kęsy, ${ }^{3}$ Kinga Lis, ${ }^{4}$ Beata Sulikowska, ${ }^{2}$ \\ Grażyna Odrowąż-Sypniewska, ${ }^{5}$ and Jacek Manitius ${ }^{2}$ \\ ${ }^{1}$ Division of Community Nursing, Faculty of Health Sciences, Ludwik Rydygier Collegium Medicum in Bydgoszcz, \\ Nicolaus Copernicus University in Toruń, Łukasiewicza 1 Street, 85-821 Bydgoszcz, Poland \\ ${ }^{2}$ Department of Nephrology, Hypertension and Internal Diseases, Faculty of Medicine, Ludwik Rydygier Collegium Medicum in \\ Bydgoszcz, Nicolaus Copernicus University in Toruń, M. Skłodowskiej-Curie 9 Street, 85-094 Bydgoszcz, Poland \\ ${ }^{3}$ Faculty of Biology and Environmental Protection, Nicolaus Copernicus University in Toruń, Lwowska 1 Street, 87-100 Toruń, Poland \\ ${ }^{4}$ Department of Allergology, Clinical Immunology and Internal Diseases, Faculty of Medicine, Ludwik Rydygier \\ Collegium Medicum in Bydgoszcz, Nicolaus Copernicus University in Toruń, Ujejskiego 75 Street, 85-168 Bydgoszcz, Poland \\ ${ }^{5}$ Department of Laboratory Medicine, Faculty of Pharmacy, Ludwik Rydygier Collegium Medicum in Bydgoszcz, \\ Nicolaus Copernicus University in Toruń, M. Skłodowskiej-Curie 9 Street, 85-094 Bydgoszcz, Poland
}

Correspondence should be addressed to Agnieszka Pluta; agnieszkapluta@poczta.onet.pl

Received 23 August 2017; Accepted 15 October 2017; Published 19 November 2017

Academic Editor: Gang Liu

Copyright (C) 2017 Agnieszka Pluta et al. This is an open access article distributed under the Creative Commons Attribution License, which permits unrestricted use, distribution, and reproduction in any medium, provided the original work is properly cited.

\begin{abstract}
Introduction. Chronic kidney disease (CKD) is accompanied by inflammation. The aim of this study was to evaluate the effect of 6-month supplementation with omega-3 acids on selected markers of inflammation in patients with CKD stages 1-3. Methods. Six-month supplementation with omega- 3 acids ( 2 g/day) was administered to 87 CKD patients and to 27 healthy individuals. At baseline and after follow-up, blood was taken for C-reactive protein (CRP) and monocyte chemotactic protein-1 (MCP1) concentration and white blood cell (WBC) count. Serum concentration of omega-3 acids-eicosapentaenoic acid (EPA), docosahexaenoic acid (DHA), and alpha-linolenic acid (ALA) - was determined using gas chromatography. And 24-hour urinary collection was performed to measure MCP-1 excretion. Results. After six-month omega-3 supplementation, ALA concentration increased in CKD patients and in the reference group, while EPA and DHA did not change. At follow-up, a significant decrease in urinary MCP-1 excretion in CKD $(p=0.0012)$ and in the reference group ( $p=0.001$ ) was found. CRP, serum MCP-1, and WBC did not change significantly. The estimated glomerular filtration rate (eGFR) did not change significantly in the CKD group. Conclusions. The reduction of urinary MCP-1 excretion in the absence of MCP-1 serum concentration may suggest a beneficial effect of omega-3 supplementation on tubular MCP-1 production. Trial Registration. This study was registered in ClinicalTrials.gov (identifier: NCT02147002).
\end{abstract}

\section{Introduction}

Chronic kidney disease (CKD) and its progression are closely linked to increased inflammatory response of the body. The severity of inflammation increases with the degree of renal failure $[1,2]$. Markers of inflammation, such as high levels of C-reactive protein (CRP), interleukin-6 (IL-6), fibrinogen, and other acute phase proteins, such as lipoprotein(a), which are also atherogenic factors, are elevated in patients with CKD $[2,3]$. On the other hand, reduced levels of proteins with antiatherogenic activity [e.g., apolipoprotein A-I and high-density lipoprotein (HDL)] are observed in CKD [3-5]. As a nonspecific marker of systemic inflammatory response, CRP activates the endothelium and accumulates in the atherosclerotic plaque, which suggests an important role in the local inflammatory process within a vessel wall $[6,7]$. It 
was confirmed that reactions associated with inflammation developing in the vessel wall are responsible for the creation and development of atherosclerotic lesions in the population of patients with CKD [8]. As regards inflammatory response in the kidneys, monocyte chemotactic protein-1 (MCP-1), belonging to the group of $\mathrm{C}-\mathrm{C}$ chemokines, cannot be overlooked. This protein is produced by many cells, particularly tubular epithelium and inflowing monocytes and macrophages [9]. In the epithelial cells of renal tubules, MCP-1 stimulates the transcription of nuclear factor-kappa B $(\mathrm{NF}-\mathrm{kB})$ and activator protein-1 (AP-1), that is, transcription factors leading to increased production of interleukin-6 (IL6) [10]. The most important task of IL-6 is to participate in the immune and inflammatory response [11]. Through extracellular signal-regulated kinase, MCP-1 induces the proliferation of vascular smooth muscle cells, contributing to the progression of renal damage [12].

Among polyunsaturated fatty acids, omega-3 or n-3 ( $\alpha$ linolenic acid, 18:3, ALA) and omega-6 or n-6 (linoleic acid, 18:2, LA) are of particular importance [13]. Several recent studies have demonstrated the beneficial effects of polyunsaturated fatty acids in reducing the inflammation and the development of atherosclerosis in the population of patients with CKD $[14,15]$. However, a different study did not confirm such a relationship [16].

The aim of this study was to evaluate the effect of 6month supplementation with omega-3 acids on selected inflammatory markers in patients with CKD stages 1-3.

\section{Methods}

The study was conducted between September 2012 and November 2014 upon approval by the Bioethics Committee of the Nicolaus Copernicus University in Torun Ludwik Rydygier Collegium Medicum in Bydgoszcz [KB 305/2012]. All patients included in the study provided written consent for participation.

The study population consisted of patients with CKD receiving treatment in the Nephrology Outpatient Clinic of University Hospital No. 1 in Bydgoszcz. The criteria for inclusion in the study were as follows: diagnosed chronic kidney disease stages 1-3, written consent from the patient to participate in the study, and age over 18 years.

Exclusion criteria included immunosuppressive therapy, diabetes, and lack of consent to participate in the study. 130 patients were asked to participate in the study; 40 refused to participate. Thirty patients without CKD, hypertension, or overt cardiovascular disease were classified as the reference group.

In the analyzed population of CKD patients, 30 patients were in stage 1, 33 in stage 2 , and 27 in stage 3 of the disease.

During the 6-month supplementation, one woman from the experimental group and one from the reference group were excluded, as they became pregnant. Two patients with CKD and two patients from the reference group failed to attend the follow-up examination after the 6-month supplementation with omega-3 acids. For the purposes of this study, the results of 87 patients with CKD and 27 patients from the reference group were analyzed both before and after supplementation.

The underlying kidney diseases in the CKD group were as follows: chronic glomerulonephritis confirmed by kidney biopsy ( $n=16 ; 18.4 \%$ ), hypertensive nephrosclerosis $(n=$ 3 ; $3.5 \%)$, polycystic kidney disease ( $n=28 ; 32.2 \%)$, gouty nephropathy $(n=5 ; 5.7 \%)$, nephrolithiasis $(n=23 ; 26.4 \%)$, and loss of one kidney due to injury $(n=1,1.1 \%)$. In 11 cases $(12.6 \%)$, the cause of the disease was unknown.

Each patient participating in the study received 6-month supplementation with omega-3 (Gold Omega 3) at a dose of 2 $\times 1000 \mathrm{mg}$. One capsule of Gold Omega $3=1000 \mathrm{mg}$ contains $65 \%$ omega- 3 acid, including $330 \mathrm{mg}$ of eicosapentaenoic acid (EPA), $220 \mathrm{mg}$ of docosahexaenoic acid (DHA), and $100 \mathrm{mg}$ of other acids including alpha-linolenic acid (ALA).

All persons participating in the study had their body mass index (BMI) calculated. Measurements were also taken of their waist and hip circumference.

Fasting blood was collected for the determination of serum creatinine, CRP, and the concentration of MCP-1. Peripheral blood leukocyte count was performed (WBC). In the 24-hour urine collection, the excretion of MCP-1 and excretion of creatinine were determined. The rate of MCP1 excretion with urine was expressed in $\mathrm{ng}$ per $1 \mathrm{mg}$ of creatinine in urine.

2.1. Laboratory Tests. Material used for the study was venous blood serum. Fasting blood was collected from the median cubital vein into two dry glass tubes without additives, in the Vacutainer closed vacuum system under standard conditions, between 7:00 and 9:00 in the morning. After collection, blood samples were left at room temperature for 30 minutes for clotting. One tube was used for the enzymatic creatinine assay using the Horiba ABX Pentra 400 biochemical analyzer. The estimated glomerular filtration rate (eGFR) was then estimated on the basis of CKD-EPI [17]. The second tube of coagulated blood was centrifuged for 15 minutes at 4000 RPM. After centrifugation, serum was separated from the blood clot. The separated serum at a volume of about $2 \mathrm{ml}$ was stored in an Eppendorf tube at $-80^{\circ} \mathrm{C}$. For the analysis of ALA, EPA, and DHA, a PerkinElmer (USA) gas chromatograph equipped with a flame ionization detector (GC-FID) was used. Separation of FAMEs was carried out on an Equity-5 (Supelco) capillary column $(30 \mathrm{~m} \times 0.25 \mathrm{~mm}$ i.d., $0.25 \mu \mathrm{m}$ film thickness) using hydrogen as the carrier gas.

$100 \mu \mathrm{l}$ of human serum was saponified in $5 \mathrm{ml}$ PTFE screw-capped glass tubes containing $10 \mu \mathrm{g}$ of tridecanoic acid as an internal standard and $1 \mathrm{ml}$ of $0.5 \%(\mathrm{w} / \mathrm{v})$ sodium methylate. The samples were heated for $15 \mathrm{~min}$ at a temperature of $100^{\circ} \mathrm{C}$ and, after cooling to room temperature, esterified with $1.5 \mathrm{ml}$ of $\mathrm{BF}_{3}$ in methanol (also at $100^{\circ} \mathrm{C}$ ) for $10 \mathrm{~min}$. Again after cooling of the tubes, $1 \mathrm{ml}$ of $n$-hexane was added to extract the fatty acid methyl esters. The contents of the tubes were then shaken for $1 \mathrm{~min}$ and $1 \mathrm{ml}$ of saturated sodium chloride solution was added. Afterwards, the tubes were centrifuged for $5 \mathrm{~min}$ at $2200 \times \mathrm{g}$. The clear hexane top layer was transferred into an injection vial, evaporated to dryness under a stream of nitrogen, and then redissolved in $100 \mu \mathrm{l}$ of hexane. $1 \mu \mathrm{l}$ of the final solution was applied into the 
GC injector. The method of FA analysis was adopted from Bondia-Pons et al. [18].

The identities of sample methyl ester peaks were determined by comparing their relative retention times with those of well-known FAME standards. Quantification was based on the amount of the internal standard recovered. The results were expressed in $\mathrm{mg} / 100 \mathrm{ml}$ of serum:

$$
\text { Concentration of } \mathrm{KT}=\frac{\mathrm{S} \mathrm{KT}}{\mathrm{S} \mathrm{ST}} \times \mathrm{M} \mathrm{ST}[\mathrm{mg} / 100 \mathrm{ml}] \text {, }
$$

where S KT is the fatty acid peak area, S ST is the internal standard peak area, and M ST is the amount of internal standard in $\mu \mathrm{g}$.

Serum levels of creatinine and C-reactive protein were determined using the Horiba ABX Pentra 400 biochemical analyzer. The number of leukocytes was determined using a conventional method.

2.2. Statistical Analysis. Distribution of variables was tested using the Shapiro-Wilk test. For comparison of results in more than two groups, the nonparametric ANOVA test was used. As a post hoc test for the detailed identification of statistically different groups, the Tukey test was used. Data were presented as mean \pm standard deviation (SD), and the median and top and bottom quartiles were given for variables that were not normally distributed. Evaluation of the correlations between the analyzed indicators was carried out using Pearson's correlation coefficient (for samples with normal distribution) and Spearman's correlation coefficient (for samples with nonnormal distribution). The level of statistical significance was set at $p<0.05$. Changes $(\Delta)$ in selected parameters were calculated. $\Delta$ was calculated by subtracting the initial value from the final value.

\section{Results}

Clinical characteristics and laboratory results in patients with CKD stages 1-3 and in the reference group before and after 6 months of supplementation with omega- 3 acid are shown in Table 1. The mean age of CKD patients was $57 \pm 11$, and in the reference group it was $54 \pm 11$. In patients with CKD and in the reference group, there was a statistically significant increase in the concentration of ALA after supplementation. Creatinine concentration and eGFR did not change in patients with $C K D$, while in the reference group, eGFR decreased and serum creatinine concentration increased. The clinical characteristics and laboratory results in the different stages of CKD are shown in Table 2.

During the supplementation with omega-3 acid, the tolerance of the Gold Omega 3 preparation was good. The occurrence of side effects in the form of belching and nausea was observed in 2 patients (2.2\%). The symptoms were transient and did not require the discontinuation of therapy. In the group of patients with CKD, a statistically significant negative correlation between eGFR and CRP $(R=-0.27$; $p=0.009$ ) was demonstrated prior to supplementation. No statistically significant correlation was found between the concentrations of EPA, DHA, and ALA and markers of inflammation. In the reference group, a statistically significant negative correlation between the concentration of ALA and the number of WBC $(R=-0.46 ; p=0.014)$ was demonstrated before supplementation. In patients with CKD, a positive correlation between serum levels of MCP-1 and CRP $(R=0.38 ; p=0.0002)$ and a negative correlation between serum levels of MCP-1 and eGFR ( $r=-0.29$; $p=$ 0.006 ) were found after supplementation.

In the entire group of patients with CKD stages 1-3, no statistically significant correlations were shown between $\Delta$ of EPA, DHA, and ALA concentration and $\Delta$ of inflammatory markers. In patients with stage $2 \mathrm{CKD}$, a statistically significant negative correlation was found between $\Delta$ of ALA concentration and $\Delta$ of 24-hour urinary excretion of MCP-1 $(r=-0.37 ; p<0.05)$.

\section{Discussion}

Irrespective of its cause, chronic kidney disease is characterized by accelerated atherosclerosis and vascular stiffening with ventricular remodeling, which increases the risk of cardiovascular events $[19,20]$. It is accompanied by inflammation, regardless of its causes, stage of disease, or age of the patient.

Recent investigations have provided more and more information on the favorable effects of omega- 3 enriched diet and omega-3 supplementation on the inflammatory process, reducing the risk of cardiovascular events in the population of patients with CKD and slowing the progression of CKD [2123]. Omega-3 fatty acids are the precursors of the synthesis of inflammatory mediators. EPA and DHA, which replace arachidonic acid in plasma membranes, reduce the amount of the substrate, out of which proinflammatory eicosanoids are formed under the influence of cyclooxygenase-2 (COX2) and lipoxygenases (LOX). Omega-3 fatty acids reduce the production of prostaglandins and 2-series thromboxane, 4-series leukotrienes, and 5-hydroxyeicosatetraenoic acid (5-HETE). An alternative substrate for COX-2 and LOX is EPA, which produces prostaglandins and thromboxane (TxA3) and consequently leads to the formation of TxA2 and 5-series leukotrienes, compounds with much weaker proinflammatory properties [22]. TxA2 enhances, among other things, the proliferation of mesangial cells in the kidney [24]. EPA and DHA are precursors of molecules responsible for the suppression of the inflammatory process. The said molecules include E-series resolvins, synthesized from EPA by LOX, as well as D-series resolvins, D-series protectins, and maresins derived from DHA [25].

The main finding of the present study was that the urinary excretion of MCP-1 in the urine decreased after 6month supplementation with omega-3 acid in both CKD and the reference group, with no change in the serum concentration of MCP-1. The largest decrease in MCP-1 in the urine was observed in patients with stage 2 CKD $(p<$ 0.001 ). This could indicate that omega- 3 acids inhibit the production of MCP- 1 by tubular epithelial cells. The beneficial effect of supplementation with omega- 3 on reducing the concentration of MCP-1 in serum has so far been revealed in only one study, which involved patients with stage $5 \mathrm{CKD}$ 
TABLE 1: Characteristics of the studied group of patients with CKD and the reference group at baseline and after supplementation with omega-3 acid.

\begin{tabular}{|c|c|c|c|c|c|c|}
\hline \multirow[t]{2}{*}{ Parameter } & \multicolumn{2}{|c|}{$\begin{array}{l}\text { Reference } \\
(n=27)\end{array}$} & \multirow{2}{*}{$\begin{array}{c}p \\
\text { (1) vs (2) }\end{array}$} & \multicolumn{2}{|c|}{$\begin{array}{c}\text { CKD } \\
(n=87)\end{array}$} & \multirow{2}{*}{$\begin{array}{c}p \\
\text { (3) versus (4) }\end{array}$} \\
\hline & Baseline (1) & Postintervention (2) & & Baseline (3) & Postintervention (4) & \\
\hline \multicolumn{7}{|l|}{ Gender } \\
\hline Women/men & $18 / 9$ & $18 / 9$ & & $40 / 47$ & $40 / 47$ & \\
\hline $\operatorname{BMI}\left(\mathrm{kg} / \mathrm{m}^{2}\right)$ & $24.5 \pm 2.9$ & $24.7 \pm 2.8$ & 0.053 & $27.2 \pm 3.9$ & $27.2 \pm 3.8$ & 0.78 \\
\hline Waist circumference $(\mathrm{cm})$ & $88.4 \pm 11.4$ & $84.7 \pm 11.4$ & 0.92 & $94.6 \pm 12.6$ & $94.4 \pm 12.4$ & 0.67 \\
\hline Hip circumference $(\mathrm{cm})$ & $101.1 \pm 5.6$ & $101.2 \pm 5.2$ & 0.94 & $105.7 \pm 7.9$ & $104.7 \pm 8.3$ & 0.016 \\
\hline Creatinine (mg/dl) & $0.75 \pm 0.20$ & $0.82 \pm 0.21$ & 0.0097 & $1.05 \pm 0.35$ & $1.12 \pm 0.46$ & 0.30 \\
\hline $\begin{array}{l}\text { eGFR CKD-EPI } \\
\left(\mathrm{ml} / \mathrm{min} / 1.73 \mathrm{~m}^{2}\right)\end{array}$ & $96.3 \pm 14.8$ & $89.9 \pm 14.9$ & 0.011 & $74.9 \pm 23.5$ & $72.3 \pm 25.5$ & 0.055 \\
\hline $\operatorname{ALA}(\mathrm{mg} / 100 \mathrm{ml})$ & $1.52(1.17 ; 2.11)$ & $2.48(2.06 ; 3.38)$ & 0.0008 & $\begin{array}{l}1.8(1.11 ; \\
2.64)\end{array}$ & $3.0(2.24 ; 3.96)$ & 0.0001 \\
\hline $\mathrm{EPA}(\mathrm{mg} / 100 \mathrm{ml})$ & $6.64 \pm 3.42$ & $7.25 \pm 3.51$ & 0.52 & $7.6 \pm 3.83$ & $8.46 \pm 4.95$ & 0.20 \\
\hline DHA (mg/100 ml) & $8.21 \pm 4.67$ & $8.31 \pm 3.36$ & 0.92 & $9.09 \pm 4.48$ & $8.95 \pm 3.84$ & 0.81 \\
\hline $\mathrm{CRP}(\mathrm{mg} / \mathrm{l})$ & $0.42(0.15 ; 1.99)$ & $0.48(0.16 ; 1.30)$ & 0.79 & $\begin{array}{l}1.03(0.25 \\
\quad 2.73)\end{array}$ & $1.17(0.40 ; 2.99)$ & 0.23 \\
\hline $\mathrm{WBC}\left(10^{3} / \mu \mathrm{l}\right)$ & $5.9 \pm 1.8$ & $5.5 \pm 1.6$ & 0.06 & $6.4 \pm 1.7$ & $6.3 \pm 1.5$ & 0.51 \\
\hline MCP-1 serum (pg/dl) & $328.4 \pm 112.4$ & $374.4 \pm 116.9$ & 0.12 & $343.2 \pm 147$ & $353.1 \pm 123.8$ & 0.45 \\
\hline $\begin{array}{l}\text { MCP-1 urinary } \\
\text { excretion (ng/24 h) }\end{array}$ & $324.8(235.7 ; 418.7)$ & 208.7 (99.9; 311.5) & 0.001 & $\begin{array}{l}427.1(284.8 \\
645.1)\end{array}$ & $299.7(155.9 ; 467.3)$ & 0.0012 \\
\hline $\begin{array}{l}\text { MCP-1 urinary } \\
\text { excretion (ng)/creatinine } \\
\text { urinary excretion (mg) }\end{array}$ & $0.411(0.276 ; 0.775)$ & $0.186(0.106 ; 0.322)$ & 0.00006 & $\begin{array}{c}0.514(0.315 \\
0.817)\end{array}$ & $0.338(0.201 ; 0.606)$ & 0.00098 \\
\hline
\end{tabular}

ALA: alpha-linolenic acid; BMI: body mass index; CRP: C-reactive protein; DHA: docosahexaenoic acid; eGFR: estimated glomerular filtration rate; EPA: eicosapentaenoic acid; MCP-1 serum: monocyte chemotactic protein 1 in serum; MCP-1 urinary excretion: 24-hour urinary excretion of monocyte chemotactic protein 1; WBC: white blood cells. MCP-1 urinary excretion/creatinine urinary excretion of creatinine: monocyte chemotactic protein 1 excretion with urine was expressed in ng per $1 \mathrm{mg}$ of creatinine in urine.

undergoing hemodialysis and receiving EPA + DHA at a dose of $2.9 \mathrm{~g} /$ day for 12 weeks. Urinary excretion of MCP-1 was not studied due to the stage of disease [26]. Experimental studies conducted on male Sprague-Dawley rats with CKD receiving omega-3 acid for 12 weeks revealed that there was a decrease in MCP-1 in serum [27]. One of the conclusions drawn by the authors was that long-term supplementation with omega3 acids attenuated tubulointerstitial fibrosis in the remnant kidney.

Inflammation of the renal interstitium increases the progression of CKD. A significant role in this respect is played by proinflammatory chemokine MCP-1, which induces the activation and migration of monocytes/macrophages, basophils, CD4 lymphocytes, and NK cells [28]. In the kidneys, MCP1 is synthesized via the activation of the NF- $\kappa \mathrm{B}$ in renal tubular epithelial cells and mesangial cells [9, 29]. Through extracellular signal-regulated kinase, MCP-1 induces the proliferation of vascular smooth muscle cells, contributing to the progression of kidney damage $[10,29,30]$. Therefore, reduced production of MCP-1 in the renal interstitium may contribute to slowing the progression of CKD by inhibiting smooth muscle proliferation. This hypothesis, however, would have to be verified by experimental studies.

The use of omega-3 acids contributes to the downward trend of daily MCP-1 urinary excretion. The largest decrease was observed in patients with CKD stage 2, who constituted the largest study group. It cannot be ruled out that if the remaining groups were bigger, a decrease in daily MCP-1 urinary excretion could be observed. In stage $2 \mathrm{CKD}$, the decrease in daily MCP-1 urinary excretion was the greatest and amounted to $42 \%$, while in CKD 3 the decrease was $28 \%$. This suggests that the beneficial effect of omega- 3 on the kidney interstitium is more favorable in the early stages of the disease due to a less advanced process of kidney interstitial fibrosis.

The present study did not reveal any effects of supplementation with omega-3 fatty acids on serum CRP levels. Similarly, no beneficial effects of fatty acids on the levels of serum CRP and IL-6 were shown in patients undergoing peritoneal dialysis and hemodialysis who received 8-week supplementation with omega- 3 acids at a dose of $3 \mathrm{~g} /$ day $[28,29,31,32]$. In a study of CKD patients with serum creatinine in the range of 150 to 400 micromoles/l after receiving 2-month supplementation with omega-3 acid at a dose of $2.4 \mathrm{~g} / \mathrm{day}$, the authors found only a tendency to reduce the concentration of hsCRP [33]. Furthermore, Deike et al., who studied a group of patients with CKD not treated with dialysis and receiving 8-week supplementation with omega-3 acid at a dose of $1.4 \mathrm{~g}$ EPA + DHA $1 \mathrm{~g} /$ day, confirmed the lack of anti-inflammatory effects of omega-3 acid [16]. 
TABLE 2: Characteristics of the studied group of patients with CKD 1, CKD 2, and CKD 3 at baseline and after supplementation with omega-3 acid.

\begin{tabular}{|c|c|c|c|c|}
\hline Parameter & $\begin{array}{c}\text { CKD 1 } \\
(n=29)\end{array}$ & $\begin{array}{c}\text { CKD 2 } \\
(n=32)\end{array}$ & $\begin{array}{l}\text { CKD 3 } \\
(n=26)\end{array}$ & $\begin{array}{c}\text { ANOVA } \\
p\end{array}$ \\
\hline \multicolumn{5}{|l|}{ Gender } \\
\hline Women/men & $14 / 15$ & $15 / 17$ & $11 / 15$ & \\
\hline \multicolumn{5}{|l|}{ Age (years) } \\
\hline Baseline & $50 \pm 11$ & $58 \pm 11^{1}$ & $63 \pm 7^{2}$ & 0.000035 \\
\hline Postintervention & $51 \pm 11$ & $59 \pm 11^{1}$ & $64 \pm 7^{2}$ & 0.000035 \\
\hline \multicolumn{5}{|l|}{ BMI $\left(\mathrm{kg} / \mathrm{m}^{2}\right)$} \\
\hline Baseline & $26.4 \pm 4.0$ & $27.6 \pm 3.9$ & $27.6 \pm 3.7$ & 0.44 \\
\hline Postintervention & $26.7 \pm 3.9$ & $27.3 \pm 3.7$ & $27.6 \pm 4.0$ & 0.67 \\
\hline \multicolumn{5}{|c|}{ Waist circumference $(\mathrm{cm})$} \\
\hline Baseline & $91.3 \pm 13.6$ & $94.6 \pm 12.5$ & $98.2 \pm 11.1$ & 0.13 \\
\hline Postintervention & $92.0 \pm 12.6$ & $93.4 \pm 11.8$ & $98.1 \pm 12.5$ & 0.17 \\
\hline \multicolumn{5}{|c|}{ Hip circumference $(\mathrm{cm})$} \\
\hline Baseline & $104.4 \pm 8.6$ & $106.4 \pm 7.4$ & $106.3 \pm 8.0$ & 0.58 \\
\hline Postintervention & $103.7 \pm 8.2$ & $105.3 \pm 7.6^{*}$ & $105.3 \pm 9.4$ & 0.70 \\
\hline \multicolumn{5}{|l|}{ Creatinine (mg/dl) } \\
\hline Baseline & $0.75 \pm 0.17$ & $1.00 \pm 0.16^{2}$ & $1.46 \pm 0.29^{1,3}$ & 0.001 \\
\hline Postintervention & $0.78 \pm 0.16$ & $1.04 \pm 0.24^{1}$ & $1.58 \pm 0.51^{1,4}$ & 0.0001 \\
\hline \multicolumn{5}{|l|}{$\begin{array}{l}\text { eGFR CKD-EPI } \\
\left(\mathrm{ml} / \mathrm{min} / 1.73 \mathrm{~m}^{2}\right)\end{array}$} \\
\hline Baseline & $101.1 \pm 9.5$ & $74.2 \pm 9.0^{2}$ & $46.6 \pm 8.1^{2,4}$ & 0.001 \\
\hline Postintervention & $97.3 \pm 12.3$ & $72 . \pm 17.2^{2}$ & $44.7 \pm 13.4^{2,4}$ & 0.001 \\
\hline \multicolumn{5}{|l|}{$\mathrm{ALA}(\mathrm{mg} / 100 \mathrm{ml})$} \\
\hline Baseline & $1.36(0.96 ; 2.47)$ & $1.98(1.48 ; 2.57)$ & $1.65(1.11 ; 2.77)$ & 0.33 \\
\hline Postintervention & $3.03(2.07 ; 3.83)^{* *}$ & $3.22(2.31 ; 4.79)^{* *}$ & $2.82(2.24 ; 3.56)^{*}$ & 0.55 \\
\hline \multicolumn{5}{|l|}{$\mathrm{EPA}(\mathrm{mg} / 100 \mathrm{ml})$} \\
\hline Baseline & $7.51 \pm 3.87$ & $8.25 \pm 4.45$ & $6.90 \pm 2.85$ & 0.42 \\
\hline Postintervention & $8.45 \pm 4.22$ & $7.81 \pm 4.21$ & $9.26 \pm 6.42$ & 0.54 \\
\hline \multicolumn{5}{|l|}{ DHA (mg/100 ml) } \\
\hline Baseline & $8.77 \pm 5.19$ & $9.37 \pm 4.25$ & $9.11 \pm 4.02$ & 0.88 \\
\hline Postintervention & $8.67 \pm 2.799$ & $8.14 \pm 3.63$ & $10.25 \pm 4.80$ & 0.10 \\
\hline \multicolumn{5}{|l|}{$\mathrm{CRP}(\mathrm{mg} / \mathrm{l})$} \\
\hline Baseline & $0.28(0.10 ; 1.06)$ & $1.67(0.51 ; 3.17)^{1}$ & $1.17(0.47 ; 2.40)^{5}$ & 0.0055 \\
\hline Postintervention & $0.77(0.35 ; 2.82)^{*}$ & $2.13(0.61 ; 3.22)$ & $0.99(0.46 ; 2.30)$ & 0.23 \\
\hline \multicolumn{5}{|l|}{$\operatorname{WBC}\left(10^{3} / \mu \mathrm{l}\right)$} \\
\hline Baseline & $6.5 \pm 1.9$ & $6.0 \pm 1.4$ & $6.7 \pm 1.7$ & 0.33 \\
\hline Postintervention & $6.2 \pm 1.7$ & $6.2 \pm 1.5$ & $6.7 \pm 1.4$ & 0.37 \\
\hline \multicolumn{5}{|l|}{ MCP-1 serum (pg/dl) } \\
\hline Baseline & $316.5 \pm 108.4$ & $370.5 \pm 158.3$ & $340.5 \pm 169.6$ & 0.37 \\
\hline Postintervention & $315.3 \pm 88.3$ & $359.1 \pm 108.6$ & $388.0 \pm 162.0^{*}$ & 0.08 \\
\hline \multicolumn{5}{|c|}{$\begin{array}{l}\text { MCP-1 urinary excretion } \\
(\mathrm{ng} / 24 \mathrm{~h})\end{array}$} \\
\hline Baseline & $362.6(275.8 ; 695.2)$ & $432.8(263.2 ; 614.1)$ & $458.4(311.6 ; 697.8)$ & 0.50 \\
\hline Postintervention & $354.63(114.8 ; 481.5)$ & $251.1(115.3 ; 437.8)^{* *}$ & $329.5(259.2 ; 468.8)$ & 0.22 \\
\hline
\end{tabular}

ALA: alpha-linolenic acid; BMI: body mass index; CRP: C-reactive protein; DHA: docosahexaenoic acid; eGFR: estimated glomerular filtration rate; EPA: eicosapentaenoic acid; MCP-1 serum: monocyte chemotactic protein 1 in serum; MCP-1 urinary excretion: 24-hour urinary excretion of monocyte chemotactic protein 1 ; WBC: white blood cells. ${ }^{*} p<0.05$ after versus before; ${ }^{* *} p<0.001$ after versus before; ${ }^{1} p<0.01$ versus CKD $1 ;{ }^{2} p<0.001$ versus CKD $1 ;{ }^{3} p<0.01$ versus CKD $2 ;{ }^{4} p<0.001$ versus CKD $2 ;{ }^{5} p<0.05$ versus CKD 1 . 


\section{Conclusions}

The reduction of urinary MCP-1 excretion in the absence of MCP-1 serum concentration may suggest a beneficial effect of omega-3 supplementation on tubular MCP-1 production. Our results suggest a potential favorable effect of omega-3 supplementation on renal interstitial inflammation.

\section{Additional Points}

Critical Comments about the Materials and Methods. The present study has several limitations. These limitations result from a relatively small study group and a short period of supplementation with omega-3 or the daily dose of omega-3 itself. Extending the observation period to up to 12 months will improve the chances of demonstrating the effect of omega-3 on the inflammatory process. The study did not include patients with CKD who did not receive supplementation with omega-3 acids and who would serve as a reference group.

\section{Disclosure}

The results of this study were presented in the form of an abstract during the 12th Session of the Polish Society of Nephrology held on 16-18 June 2016 in Łódź.

\section{Conflicts of Interest}

The authors declare that they have no conflicts of interest.

\section{Acknowledgments}

The authors would like to thank the Company Olimp Laboratories Sp. z o.o. which provided the Gold Omega 3 preparation for supplementation for a period of 6 months. This paper was supported by the "NERKADAR" foundation at the Department of Nephrology, Hypertension and Internal Medicine of NCU Collegium Medicum in Bydgoszcz.

\section{References}

[1] F. Locatelli, B. Canaud, K.-U. Eckardt, P. Stenvinkel, C. Wanner, and C. Zoccali, "Oxidative stress in end-stage renal disease: an emerging treat to patient outcome," Nephrology Dialysis Transplantation, vol. 18, no. 7, pp. 1272-1280, 2003.

[2] R. L. Amdur, H. I. Feldman, J. Gupta et al., "Inflammation and Progression of CKD: The CRIC Study," Clinical Journal of the American Society of Nephrology, vol. 11, no. 9, pp. 1546-1556, 2016.

[3] F. C. Amparo, M. A. Kamimura, M. Z. Molnar et al., "Diagnostic validation and prognostic significance of the MalnutritionInflammation Score in nondialyzed chronic kidney disease patients," Nephrology Dialysis Transplantation, vol. 30, no. 5, pp. 821-828, 2015.

[4] N. D. Vaziri, "Lipotoxicity and impaired high density lipoprotein-mediated reverse cholesterol transport in chronic kidney disease," Journal of Renal Nutrition, vol. 20, no. 5, supplement, pp. S35-S43, 2010.
[5] S. Swaminathan and S. V. Shah, "Novel inflammatory mechanisms of accelerated atherosclerosis in kidney disease," Kidney International, vol. 80, no. 5, pp. 453-463, 2011.

[6] V. Pasceri, J. T. Willerson, and E. T. H. Yeh, "Direct proinflammatory effect of C-reactive protein on human endothelial cells," Circulation, vol. 102, no. 18, pp. 2165-2168, 2000.

[7] C. D. A. Stehouwer, "Endothelial dysfunction in diabetic nephropathy: State of the art and potential significance for nondiabetic renal disease," Nephrology Dialysis Transplantation, vol. 19, no. 4, pp. 778-781, 2004.

[8] R. Pecoits-Filho, P. Bárány, B. Lindholm, O. Heimbürger, and P. Stenvinkel, "Interleukin-6 is an independent predictor of mortality in patients starting dialysis treatment," Nephrology Dialysis Transplantation, vol. 17, no. 9, pp. 1684-1688, 2002.

[9] H. R. Vianna, C. M. B. M. Soares, K. D. Silveira et al., "Cytokines in chronic kidney disease: Potential link of MCP-1 and dyslipidemia in glomerular diseases," Pediatric Nephrology, vol. 28, no. 3, pp. 463-469, 2013.

[10] C. Viedt and S. R. Orth, "Monocyte chemoattractant protein1 (MCP-1) in the kidney: does it more than simply attract monocytes?" Nephrology Dialysis Transplantation, vol. 17, no. 12, pp. 2043-2047, 2002.

[11] T. Kishimoto, "Interleukin-6: discovery of pleiotropic cytokine," Arthritis Research \& Therapy, vol. 8, supplement 2, article S2, 2006.

[12] C. Viedt, J. Vogel, T. Athanasiou et al., "Monocyte chemoattractant protein-1 induces proliferation and interleukin-6 production in human smooth muscle cells by differential activation of nuclear factor- $\kappa \mathrm{B}$ and activator protein-1," Arteriosclerosis, Thrombosis, and Vascular Biology, vol. 22, no. 6, pp. 914-920, 2002.

[13] P. Sicińska, E. Pytel, J. Kurowska, and M. Koter-Michalak, "Supplementation with omega fatty acids in various diseases," Postepy Higieny i Medycyny Doswiadczalnej, vol. 69, pp. 838852, 2015.

[14] R. G. Fassett, G. C. Gobe, J. M. Peake, and J. S. Coombes, "Omega-3 polyunsaturated fatty acids in the treatment of kidney disease," American Journal of Kidney Diseases, vol. 56, no. 4, pp. 728-742, 2010.

[15] S. M. Lee and W. S. An, "Cardioprotective effects of $\omega$-3 PUFAs in chronic kidney disease," BioMed Research International, vol. 2013, Article ID 712949, 8 pages, 2013.

[16] E. Deike, R. G. Bowden, J. J. Moreillon et al., "The effects of fish oil supplementation on markers of inflammation in chronic kidney disease patients," Journal of Renal Nutrition, vol. 22, no. 6, pp. 572-577, 2012.

[17] A. S. Levey, L. A. Stevens, C. H. Schmid et al., "A new equation to estimate glomerular filtration rate," Annals of Internal Medicine, vol. 150, no. 9, pp. 604-612, 2009.

[18] I. Bondia-Pons, A. I. Castellote, and M. C. López-Sabater, "Comparison of conventional and fast gas chromatography in human plasma fatty acid determination," Journal of Chromatography B, vol. 809, no. 2, pp. 339-344, 2004.

[19] A. Gluba-Brzózka, M. Michalska-Kasiczak, B. Franczyk et al., "Markers of increased atherosclerotic risk in patients with chronic kidney disease: a preliminary study," Lipids in Health and Disease, vol. 15, no. 1, article 32, 2016.

[20] A. Pluta, P. Strózecki, M. Krintus, G. Odrowąz-Sypniewska, and J. Manitius, "Left ventricular remodeling and arterial remodeling in patients with chronic kidney disease stage 1-3," Renal Failure, vol. 37, no. 7, pp. 1105-1110, 2015. 
[21] X. Huang, B. Lindholm, P. Stenvinkel, and J. J. Carrero, "Dietary fat modification in patients with chronic kidney disease: N-3 fatty acids and beyond," Journal of Nephrology, vol. 26, no. 6, pp. 960-974, 2013.

[22] H. M. Aukema, "Lipids in chronic kidney disease: Alterations and interventions," Lipid Technology, vol. 25, no. 9, pp. 207-209, 2013.

[23] F. Lauretani, M. Maggio, F. Pizzarelli et al., "Omega-3 and renal function in older adults," Current Pharmaceutical Design, vol. 15, no. 36, pp. 4149-4156, 2009.

[24] S. Castellani, R. Paniccia, C. Di Serio et al., "Thromboxane inhibition improves renal perfusion and excretory function in severe congestive heart failure," Journal of the American College of Cardiology, vol. 42, no. 1, pp. 133-139, 2003.

[25] J. Z. Nowak, "Anti-inflammatory pro-resolving derivatives of omega-3 and omega-6 polyunsaturated fatty acids," Postepy Higieny i Medycyny Doswiadczalnej, vol. 64, pp. 115-132, 2010.

[26] A. M. Hung, C. Booker, C. D. Ellis et al., "Omega-3 fatty acids inhibit the up-regulation of endothelial chemokines in maintenance hemodialysis patients," Nephrology Dialysis Transplantation, vol. 30, no. 2, pp. 266-274, 2015.

[27] W. S. An, H. J. Kim, K.-H. Cho, and N. D. Vaziri, “Omega-3 fatty acid supplementation attenuates oxidative stress, inflammation, and tubulointerstitial fibrosis in the remnant kidney," American Journal of Physiology-Renal Physiology, vol. 297, no. 4, pp. F895F903, 2009.

[28] H. Tayyebi-Khosroshahi, J. Houshyar, R. Dehgan-Hesari et al., "Effect of treatment with omega-3 fatty acids on C-reactive protein and tumor necrosis factor-alfa in hemodialysis patients," Saudi Journal of Kidney Diseases and Transplantation, vol. 23, no. 3, pp. 500-506, 2012.

[29] S. Giunti, G. H. Tesch, S. Pinach et al., "Monocyte chemoattractant protein-1 has prosclerotic effects both in a mouse model of experimental diabetes and in vitro in human mesangial cells," Diabetologia, vol. 51, no. 1, pp. 198-207, 2008.

[30] H. Haller, A. Bertram, F. Nadrowitz, and J. Menne, "Monocyte chemoattractant protein-1 and the kidney," Current Opinion in Nephrology and Hypertension, vol. 25, no. 1, pp. 42-49, 2016.

[31] S. L. Deshmane, S. Kremlev, S. Amini et al., "Monocyte Chemoattractant Protein-1 (MCP-1): an overview," Journal of Interferon \& Cytokine Research, vol. 29, no. 6, pp. 313-326, 2009.

[32] A. E. Naini, R. E. Asiabi, N. Keivandarian, and F. Moeinzadeh, "Effect of omega-3 supplementation on inflammatory parameters in patients on chronic ambulatory peritoneal dialysis," Advanced Biomedical Research, vol. 10, no. 4, pp. 167-172, 2015.

[33] T. Madsen, E. B. Schmidt, and J. H. Christensen, "The effect of n3 fatty acids on C-reactive protein levels in patients with chronic renal failure," Journal of Renal Nutrition, vol. 17, no. 4, pp. 258$263,2007$. 


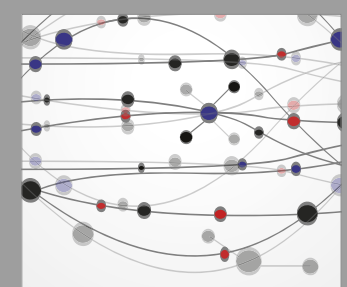

The Scientific World Journal
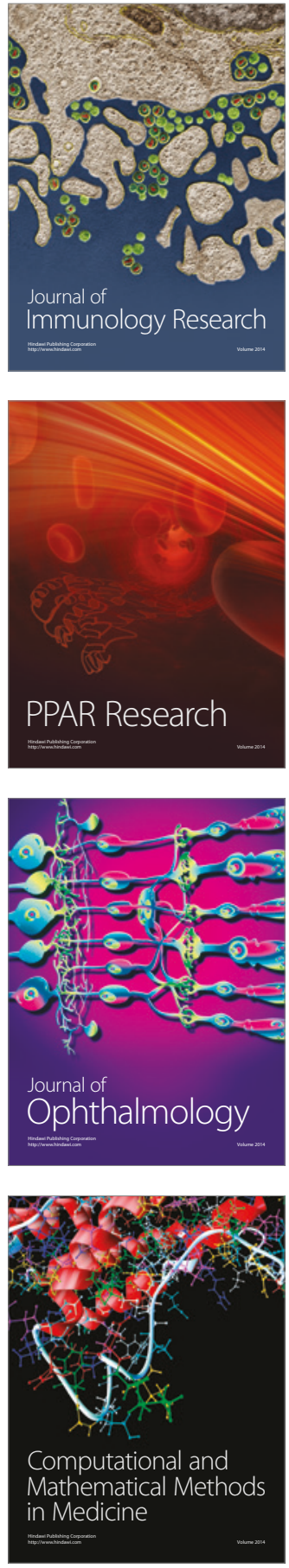

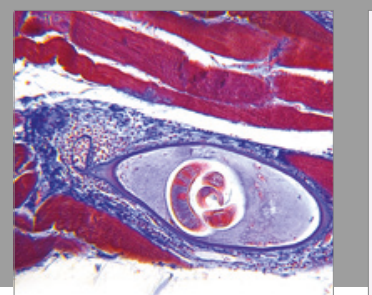

Gastroenterology Research and Practice
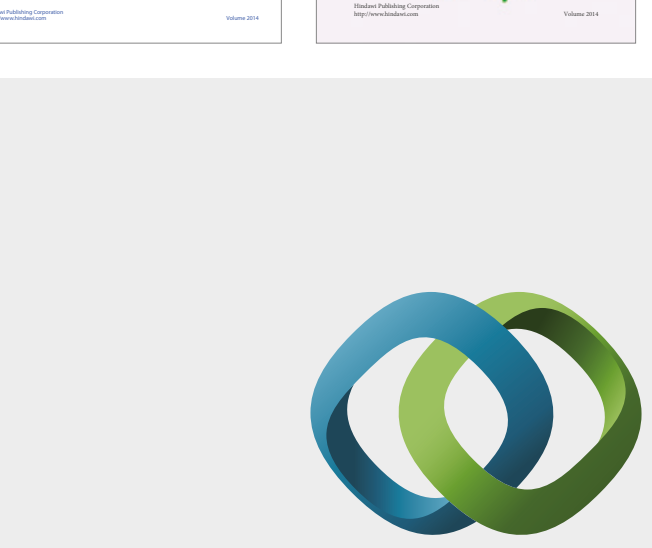

\section{Hindawi}

Submit your manuscripts at

https://www.hindawi.com
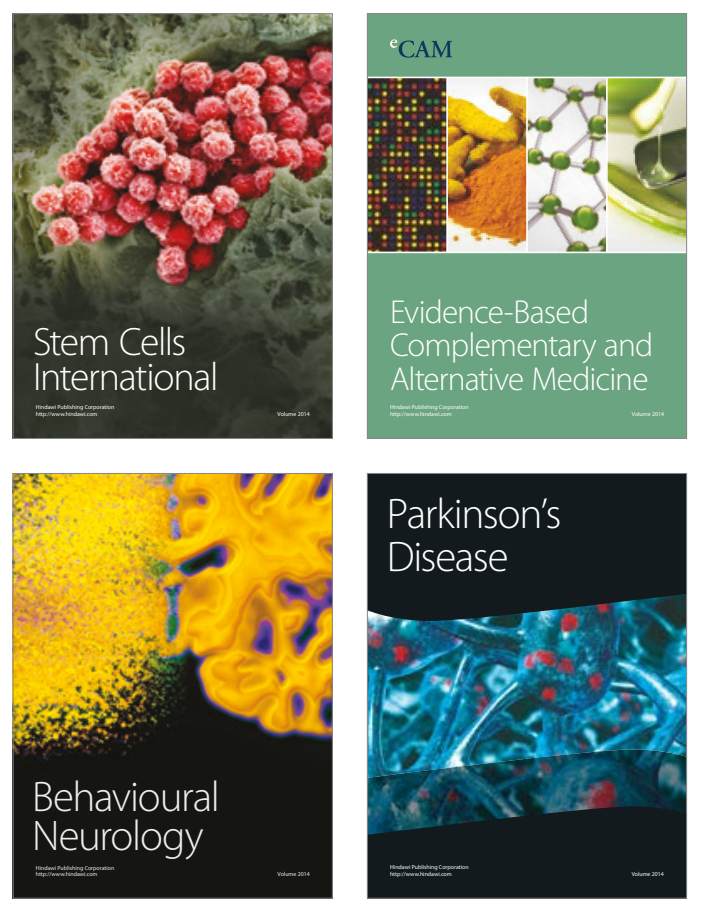
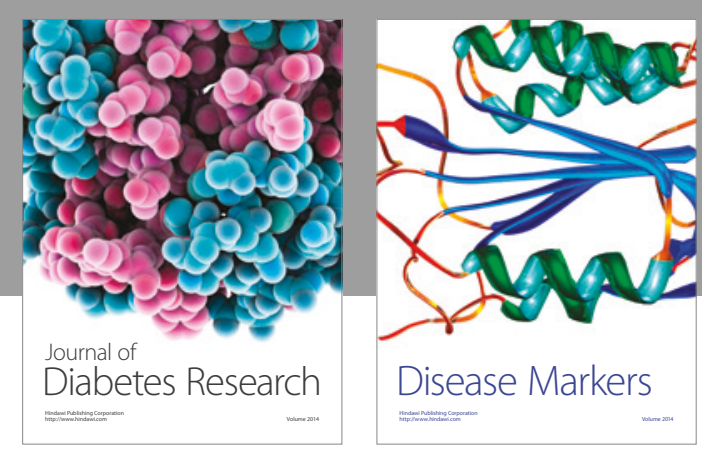

Disease Markers
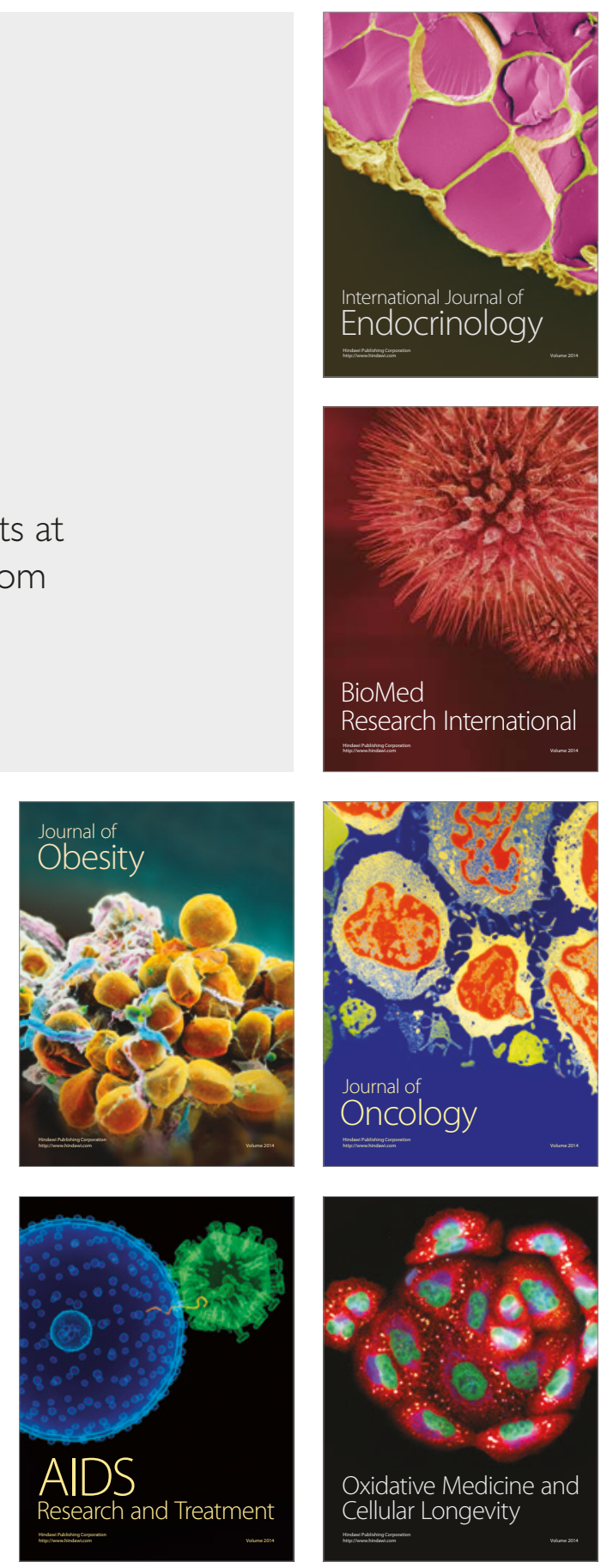